\title{
Fate of dura mater homografts patching superior vena caval defects ${ }^{1}$
}

\author{
PANAGIOTIS C. PETROPOULOS \\ From the Department of Cardiovascular Surgery, Presbyterian Medical Center, San Francisco, Calif.
}

Following the work by Gerbode, Yee, and Rundle (1949) concerning successful atriocaval anastomosis, many experimental and clinical reports have emerged reporting the results of patching defects or replacing segments of the superior vena cava. Autologous (Deterling and Bhonslay, 1955), homologous (Ashburn, Sewell, and Huggins, 1956; Holman and Steinberg, 1954), heterologous, and plastic grafts (Collins, Burrus, and DeBakey, 1960; Riberi, Pompey, and Hritzo, 1961) have been used, but dura mater homografts have not, until this present study, been applied to this particular problem.

\section{METHOD}

Ten mongrel dogs weighing from 13 to $18 \mathrm{~kg}$. were used. Anaesthesia was induced and continued with Nembutal, and cyclic positive pressure insufflation of the lungs with room air was maintained. The thorax was opened through the right third interspace and the superior vena cava mobilized. After control of the dissected cava with occlusive clamps an oval defect of the anterior wall of the caval vein, $2 \times 2 \mathrm{~cm}$., was created at the level of the azygos vein. The dura mater homografts used were either fresh or preserved in the refrigerator from one to eight days in a saline-penicillin-streptomycin solution, and were so shaped as to be a little larger than the defect. The soft and pliable dural patch was sutured to the edges of the defect with 6-0 silk everting sutures without undue tension. The distal caval clamp was then released and any trapped air was expressed through the interstices. The occlusion period was about 10 minutes, and at the end of the operation superior vena caval patency was adequate in all the animals. Significant spasm of the vein occurred three times; this did not relax until the chest was closed. No anticoagulants were administered postoperatively.

\footnotetext{
1 Supported by funds from the U.S. Public Health Service. 2 Fellow in cardiovascular surgery, Stanford University Medical School, Palo Alto, Calif., and the Institute of Medical Sciences, Presbyterian Medical Center, San Francisco, Calif.
}

RESULTS

Eight dogs survived and two died on the eleventh and twenty-first post-operative days from pulmonary infection and empyema. Pathological examination of the grafted veins of the dead animals revealed neither stricture nor thrombus formation, the lumen of the cava being patent and the dural patch endothelialized. Histologically the central portion of the graft was replaced by connective tissue. At the periphery of the graft the structure of the dura was still recognizable.

The dogs that survived were in good health. Their appetites were good and their activity was not restricted. Retrograde venograms of the superior vena cava were performed between the fourth and the tenth post-operative months. This was done by injecting Hypaque into the right atrium through a catheter passed via the femoral vein. Patency of the superior vena cava, without narrowing at the grafted area, was found in seven dogs (Fig. 1). In one dog a slight constriction of $2 \mathrm{~mm}$. was evident in this region. Dilatation of the vein was not seen.

The dogs were sacrificed between the fourth and tenth post-operative months. Adhesions of the surrounding lung to the patch region were found in five dogs. These adhesions were fibrous in one dog and were associated with stenosis of the lumen of the vein. In the remaining seven dogs the lumen of the vena cava was normal. The external surface of the grafted region was smooth without perivascular scars (Fig. 2). Thrombus formation in the lumen was not observed. Histologically the graft was not recognizable as dura mater four months after implantation, having been replaced by connective tissue. A layer of apparently normal endothelial cells covered the internal surface of the patch (Fig. 3).

\section{DISCUSSION}

Despite the great variety of grafts used to replace caval veins, satisfactory results have not been achieved. Most reports indicate that when arterial 


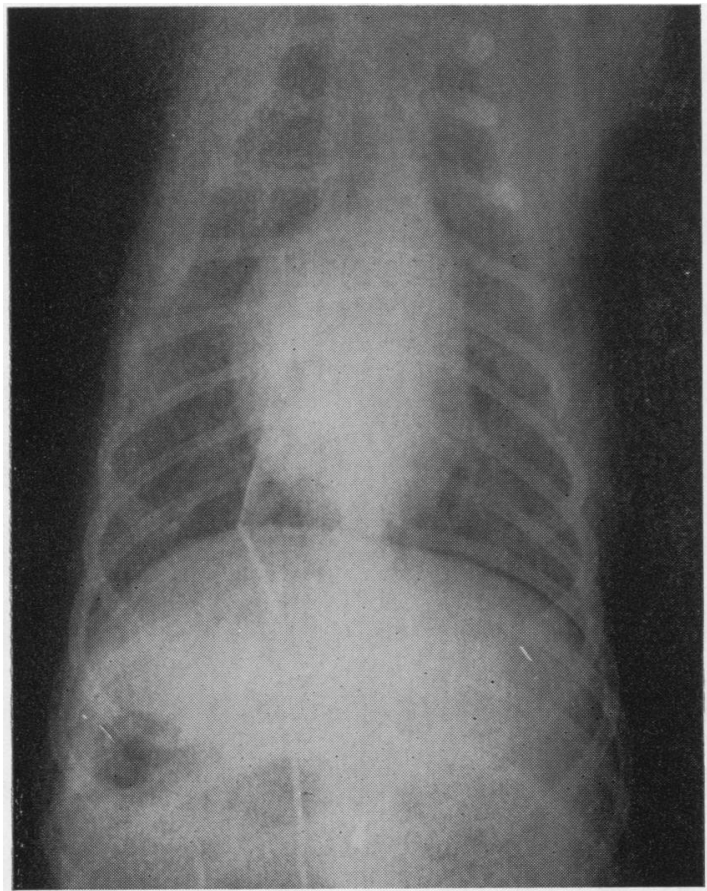

FIG. 1

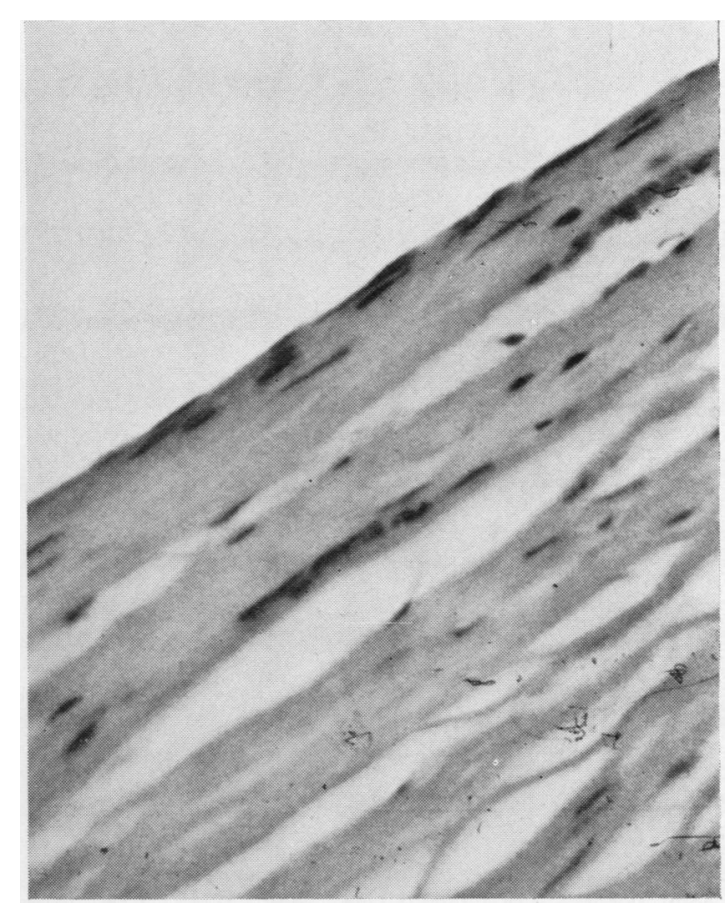

FIG. 3

FIG. 1. Complete patency of the superior vena cava eight months after replacement of a defect of its wall with a dural patch.

FIG. 3. A layer of endothelial cells covers the luminal surface of the graft, $\times 80$.

(Ashburn et al., 1956; Holman and Steinberg, 1954), venous (Deterling and Bhonslay, 1955), pericardial, or peritoneal autografts or homografts, or Dacron (Collins et al., 1960) or Teflon (East and Muller, 1960) grafts were used to bridge superior vena caval

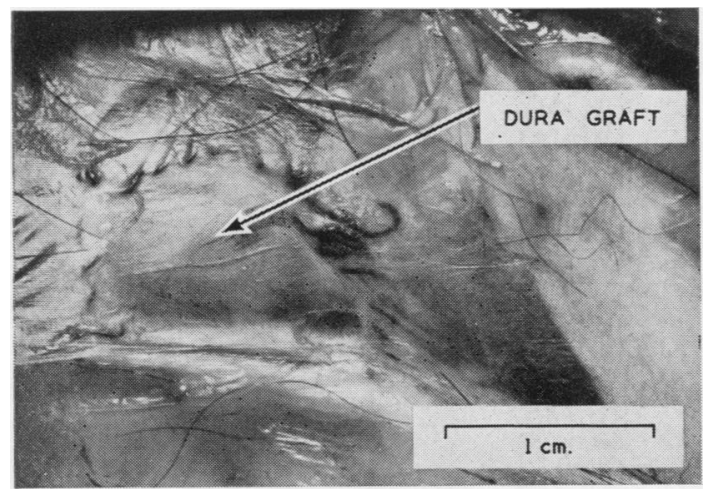

FIG. 2. The external surface of the graft is smooth without any retraction. defects, thrombosis and contraction followed with reduction or complete occlusion of the lumen. However, Moore and Riberi (1958) reported successful results when autogenous aorta was used as the graft. Recently, Riberi and his associates (1961) have replaced defects created in the superior vena cava with fine nylon mesh, and the results were satisfactory in each instance with neither thrombosis nor narrowing. The closure of the anterior wall of vena caval defects with dural patch grafts is technically easy. This is due, in part, to the softness and pliability of the dura mater which is resistant to tears in any axis and holds sutures well because its collagen fibres are placed at right angles (Usher, 1957 ; 1958). It is interesting that the fate of dural homografts is not the same when they are used to replace segments of the wall of heart cavities containing venous blood at low pressure. In previous experiments, for example, we showed that dural homografts replacing the anterior wall of the right atrium contracted (Petropoulos, in preparation). Such late contraction did not occur when dura was used to replace defects in the superior vena cava. 
Dura mater homograft patches were used to replace defects of the anterior wall of the superior vena cava. Perfect patency was present in seven out of eight long-term survivors. In the remaining dog, a small constriction of the lumen to $2 \mathrm{~mm}$. occurred. Endothelization of the graft was accomplished within two weeks of implantation. Thrombosis of the veins has not been observed.
Ashburn, F. S., Sewell, W. H., and Huggins, C. E. (1956). J. thorac. Surg., 31, 618.

Collins, H. A., Burrus, G., and DeBakey, M. E. (1960). Amer. J. Surg., 99, 40.

Deterling, R. A., and Bhonslay, S. B. (1955). Surgery, 38, 1008

East, W. M., and Muller, W. H. (1960). Amer. J. Surg., $99,6$.

Gerbode, F., Yee, J., and Rundle, F. F. (1949). Surgery, 25, 556

Holman C. W., and Steinberg I (1954) J Amer med. Ass. 155, 1403 $\mathcal{C}$

Holman, C. W., and Steinberg, 1. (1954). J. Amer. med. Ass. Moore, T. C., and Riberi, A. (1958). Surgery, 44, 898.
Riberi, A., Pompey, D. T., and Hritzo, R. E. (1961). J. thorac. Surg., $\vec{\circ}$
41, 802.

Usher, F. C. (1957). Amer. Surgn., 23, 281.

(1958). A.M.A. Arch. Surg., 76, 58. 